\title{
Broken affordances, broken objects: A TMS study
}

\author{
Giovanni Buccino ${ }^{\mathrm{a}}$, Marc Sato ${ }^{\mathrm{b}}$, Luigi Cattaneo ${ }^{\mathrm{c}}$, Francesca Rodà ${ }^{\mathrm{c}}$, Lucia Riggio $^{\mathrm{c}, *}$ \\ a Dipartimento di Scienze Mediche, Università Magna Graecia di Catanzaro, Italy \\ ${ }^{\mathrm{b}}$ GIPSA-LAB, Département Parole et Cognition, Grenoble Universités, France \\ ${ }^{\mathrm{c}}$ Dipartimento di Neuroscienze, Università di Parma, Italy
}

\section{A R T I C L E I N F O}

\section{Article history:}

Received 24 March 2009

Received in revised form 30 June 2009

Accepted 7 July 2009

Available online 15 July 2009

\section{Keywords:}

Pragmatic features

Premotor cortex

Dorsal stream

TMS

\begin{abstract}
A B S T R A C T
It is well known that specific components of motor programs are automatically activated when they are afforded by object related pragmatic features. Among these features the handle appears to be particularly salient for interacting with an object. The aim of this study was to test the modulation of the motor system when object features, particularly relevant for the action, like the object's handle, are violated. In order to address this issue a TMS paradigm was used in which familiar objects with a whole or broken handle, positioned to the right or to the left, were centrally presented. A control condition was also included in which a symbol ('\#' character) was shown in the right or in the left visual field. Participants had to watch stimuli carefully. The left hemisphere hand motor area was magnetically stimulated $200 \mathrm{~ms}$ after stimulus presentation. Results showed that MEP areas were larger when the handle was located to the right side consistent with the visuomotor role of this feature, but only when the handle was complete. The present data (1) suggest a more active role of the dorsal stream in building up object knowledge and (2) allow one to rule out the role of any asymmetrical aspect of an object in motor coding.
\end{abstract}

(c) 2009 Elsevier Ltd. All rights reserved.

\section{Introduction}

When interacting with the environment, appropriate behaviour requires a functional link between perceptual and motor systems. The concept of affordance, first introduced by Gibson (1977, 1979/1986), refers to the properties of a surface or an object in the environment, that potentiate within a perceiver, specific actions upon it. Consistent with this view, recent behavioural and neurophysiological studies demonstrate that the mere observation of an object involves accessing motor programs for interaction with the object, even in the absence of explicit intentions to act. More specifically, it has been shown that pragmatic features of an object automatically trigger components of specific actions, such as reaching or grasping (Craighero, Fadiga, Rizzolatti, \& Umiltà, 1999; Ellis \& Tucker, 2000; Phillips \& Ward, 2002; Tucker \& Ellis, 1998, 2001, 2004). Among these object features, the handle appears to be particularly salient for interaction. In fact, even if a cup can be grasped from the top or the body, there is no doubt that the handle is the component that most commonly triggers the coherent motor program to reach the cup, grasp it and eventually bring it to the mouth. In keeping with this, Tucker and Ellis (1998) showed that the orientation of the handle (right or left), although irrelevant

\footnotetext{
* Corresponding author at: Dipartimento di Neuroscienze, Università di Parma, Via Volturno 39, 43100 Parma, Italy. Tel.: +39 0521 033946; fax: +39 0521033900. E-mail address: riggio@unipr.it (L. Riggio).
}

for the task, lead to faster responses when the hand was ipsilateral to the position of the handle. Similarly, Phillips and Ward (2002) showed that in a paradigm in which the target was superimposed on an object, the handle of the object being oriented to the right, left or in a neutral position, participants were faster when the handle orientation was coherent with the response position.

These behavioural results have their counterpart in neurophysiological studies showing that, both in humans and in monkeys, neural circuits involving sectors of the parietal and premotor cortex are devoted to coding the pragmatic features of objects. In the monkey, within an area inside the intraparietal sulcus (AIP), neurons are endowed with visual and motor properties like the shape, size and orientation of a specific observed object (Murata et al., 1997; Sakata, Taira, Murata, \& Mine, 1995). In a sector of the premotor cortex (area F5) neurons which discharge during the execution of specific goal directed actions like grasping, holding, manipulating specific objects, have been found (Rizzolatti et al., 1988). A set of F5 neurons also have visual features and selectively respond to the observation of objects with pragmatic features coherent with the action motorically coded by the neuron (canonical neurons). It has been hypothesised that AIP and F5 interact strictly in affording the most suitable motor program for acting upon an object, when selected (Jeannerod, 1995).

As for humans, in an early PET study Grafton, Fadiga, Arbib, and Rizzolatti (1997) showed that the observation of common objects leads to the activation of the left premotor cortex thus implying an 
automatic recruitment of the motor system during object observation, even in the absence of any motor output. In a further fMRI study, Binkofski et al. (1999) showed that the manipulation of an object recruits brain regions in humans including the ventral premotor cortex, the inferior frontal gyrus, and an area inside the intraparietal sulcus, the latter two areas possibly corresponding to the human homologues of F5 and AIP, respectively. In keeping with these results, Chao and Martin (2000) also identified a frontoparietal circuit during the observation of pictures showing tools. Grèzes, Tucker, Armony, Ellis, and Passingham (2003) showed that the degree of activation within the fronto-parietal circuit during the execution of power or precision grip responses covaried with the action afforded by the object.

This experimental evidence fits the well-known theoretical model introduced by Milner and Goodale (1995) which claimed that visual input is segregated into two main pathways: a ventral stream and a dorsal stream, each responsible for a different analysis of visual information. According to this model, the ventral stream (from occipital visual areas to temporal lobe) is mainly involved in processing semantic knowledge of visually presented objects, while the dorsal stream (from the occipital visual areas to premotor cortex through the parietal lobe) is responsible for visuomotor transformations necessary to act (for a review, see Milner \& Goodale, 2008). The dorsal stream appears to have a specific role in on-line control during the course of an action. Fischer and Dahl (2007) showed observers an animation of a rotating cup and found spontaneous lateralized motor preparation more pronounced for the right than the left-hand, thus showing that vision for action is rapidly updated. This raises the issue of how the motor system is modulated when the most important features of an object relevant for action are violated. In other words what happens in our motor system when we look at a cup with a broken handle? Given the privileged role of some object components in affecting the motor response and the role of these in visuomotor transformations, we could expect that the motor programs triggered by the handle are violated as well. Since the excitability of primary motor cortex reflects the activity of the premotor-parietal circuits known to be involved in sensorimotor transformations (Rizzolatti \& Luppino, 2001), we employed single pulse TMS technique to address this issue.

\section{Methods}

\subsection{Participants}

Twelve subjects (seven males and five females; mean age $\pm S D, 25 \pm 4$ years) participated in the experiment. All were right-handed, according to a standard handedness inventory (Oldfield, 1971) and had normal or corrected-to-normal vision. Participants were screened for neurological, psychiatric, and other medical problems, and also for any contraindications to TMS (Wassermann, 1998). Informed consent was obtained from all subjects. Participants were compensated for their time. The protocol was approved by the Parma University Ethical Committee and was carried out in accordance with the ethical standards of the Declaration of Helsinki (1964).

\subsection{Electromyography}

Electromyography (EMG) recordings (pre-stimulus record, $200 \mathrm{~ms}$; poststimulus record, $100 \mathrm{~ms}$ ) from the right opponens pollicis (OP) and first dorsalis interosseous (FDI) hand muscles were acquired with a CED Micro 1401 analogto-digital converting unit (Cambridge Electronic Design, Cambridge, UK). The EMG signal was amplified $(1000 \times)$ and digitized (sampling rate: $8 \mathrm{kHz}$, bandpass filter: $60-4000 \mathrm{~Hz}$ ) and stored on a computer for offline analysis. The $\mathrm{OP}$ and FDI muscle activity was recorded from the right-hand using $\mathrm{Ag} / \mathrm{AgCl}$ surface electrodes with a bipolar montage. The active electrode was placed on the muscle belly and the reference electrode on the corresponding tendon.

\subsection{Transcranial magnetic stimulation}

The left hemisphere was magnetically stimulated by means of monophasic single pulses delivered through a figure-eight coil connected to a transcranial magnetic stimulator (ESAOTE Biomedica, Italy). Using previous studies carried out in our lab
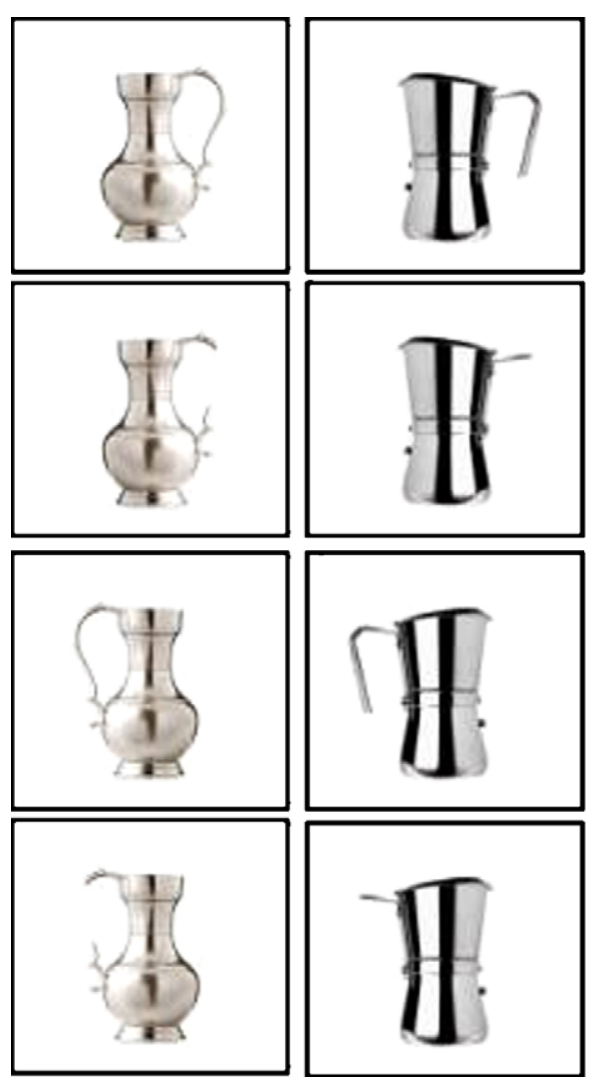
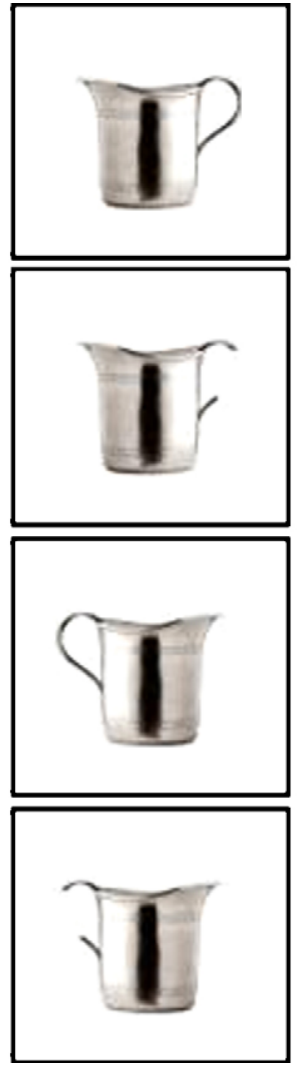
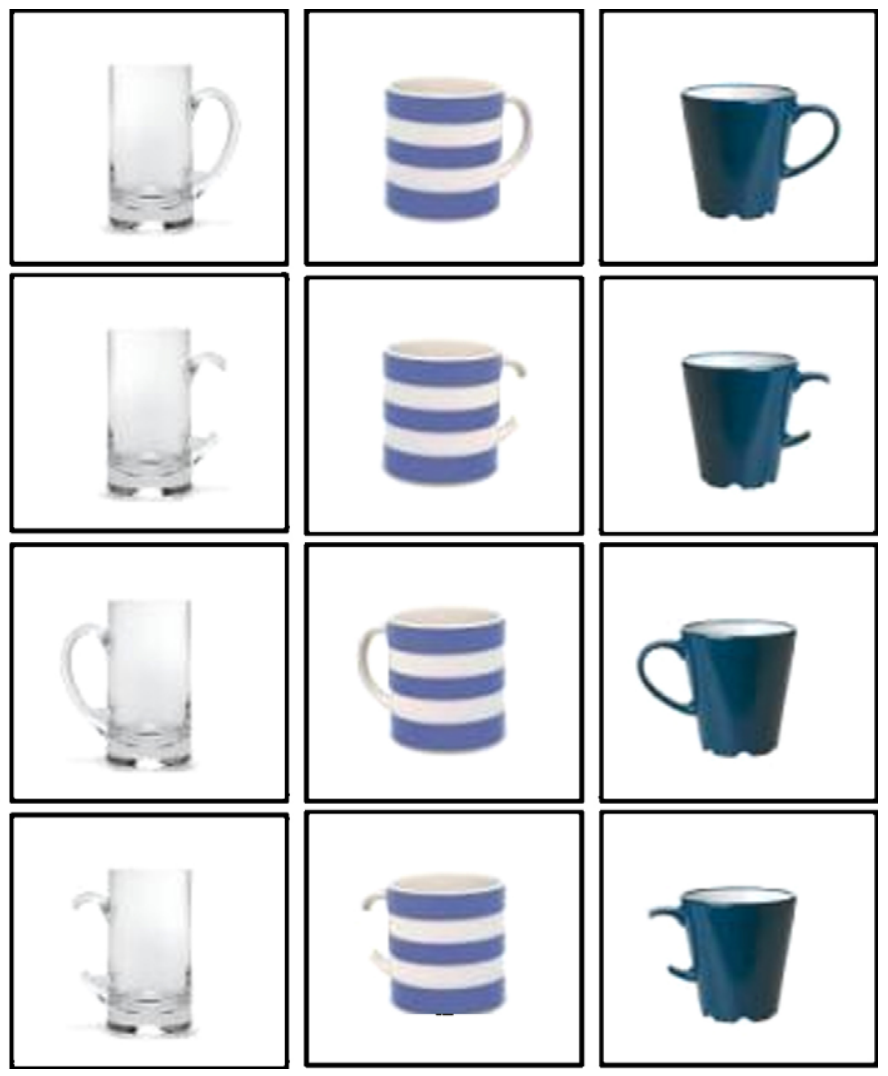

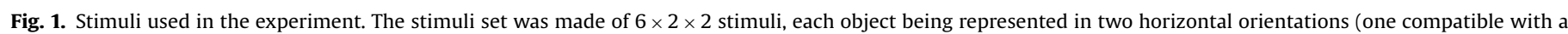
right-hand grasp, the other with a left-hand grasp) and according to two different types of handle (one normal and compatible with a hand grasp, the other broken). 


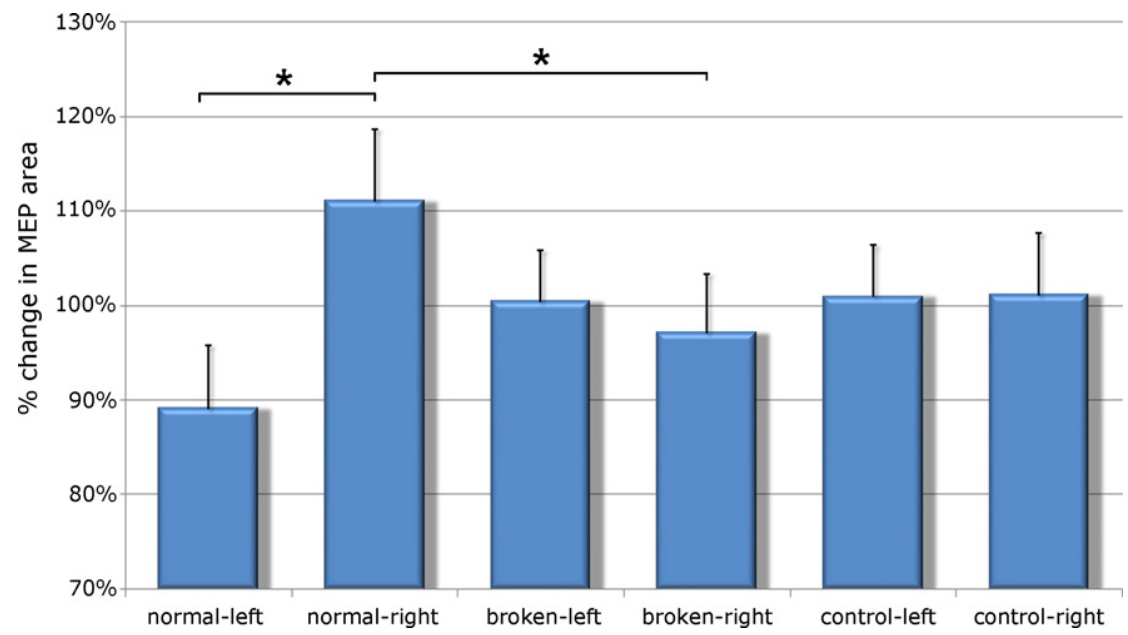

Fig. 2. Mean MEP areas per condition expressed as a percentage of the grand mean. Error bars represent standard errors of the mean.

as a reference (where hand motor representation was located 5-6 cm laterally and 1-2 cm anterior from the vertex) we determined for each participant the best site from which maximal amplitude motor-evoked potentials (MEPs) could be elicited in both the OP and FDI muscles, moving the coil over the scalp on fixed positions of a grid, with a resolution of $1 \mathrm{~cm}$. The coil was placed tangentially to the scalp with the handle pointing backward and laterally at a $45^{\circ}$ angle away from the midline (Mills, Boniface, \& Schubert, 1992). After determining the best site of stimulation, the coil handle was fixed in a mechanical arm, in order to suppress movements of the coil from the original position on the scalp. The resting motor thresholds (rMThs) of OP and FDI muscles were determined, according to standard methods, as the minimal stimulation intensity capable of evoking MEPs with an amplitude of at least $50 \mu \mathrm{V}$ in relaxed muscle in 5 out of 10 consecutive trials (Rossini et al., 1994). A single optimal spot on the scalp was found allowing MEPs to be evoked for the two muscles with similar thresholds. In this way, no individual differences in rMTh between the two muscles were found in any subject. Stimulation intensity during the experimental session was $120 \%$ of the rMTh. Complete muscle relaxation before TMS was verified by means of on-line visual monitoring of the EMG signal by the experimenter

\subsection{Stimuli}

Stimuli consisted of normal objects with a whole handle (normal affordance) or with a broken handle (broken affordance), or of the '\#' symbol (absent affordance). Colour digital photographs $(500 \times 450$ pixels $)$ of 6 common containers were selected (see Fig. 1). All objects had a handle that could be grasped and manipulated with one hand. Each object was presented twice with the handle oriented either to the right or the left side; the same objects were also presented twice with a broken handle. In addition, the "\#" symbol was presented 12 times in either the left or the right side of the screen. Therefore, for each stimulus type (normal, broken and absent affordance) and side (left, right), 12 trials were presented. A unique randomization of the 72 trials was created for each participant.

\subsection{Procedure}

The experiment was implemented using Matlab (The Mathworks Inc., Natick, MA), Cogent (Functional Imaging Laboratory, Queen Square, London, UK) and Signal (Cambridge Electronic Design, Cambridge, UK) softwares to present stimuli, to deliver TMS pulses and to record MEPs, respectively.

Participants were seated comfortably on an armchair with the right elbow flexed at $90^{\circ}$ and the right-hand maintained in a totally relaxed position. The participant's head was supported by a headrest to maintain a comfortable and stable position in front of a $19 \mathrm{in}$. monitor which was positioned at approximately $60 \mathrm{~cm}$ distance. Participants were required to watch stimuli carefully. Stimuli were presented at the centre of the screen.

Each trial started with a fixation point (the '+' symbol). After $500 \mathrm{~ms}$, the fixation point was replaced by a stimulus for $500 \mathrm{~ms}$ (normal object, broken object, or symbol). In the trials in which no object was presented, the fixation point remained along with the stimulus for $500 \mathrm{~ms}$. A TMS pulse was delivered $200 \mathrm{~ms}$ after stimulus presentation. Stimulus presentation was followed by a blankscreen of $4000 \mathrm{~ms}$ duration. In order to check that participants were paying attention to the task, 12 randomly run trials were added, in which the name of one of the six objects was visually presented. In these trials, participants were required to decide whether or not the name of the object corresponded to the stimulus presented in the previous trial, and to give a coherent oral response ('yes'/'no'). No TMS pulse was delivered during these trials.

\subsection{Data analysis}

Data were processed offline. The $200 \mathrm{~ms}$ pre-stimulus recordings were inspected to assess the possible presence of EMG activity before TMS. Trials with EMG activity before TMS were removed from the analysis (on average 4\% of all trials). For each participant, each muscle and each trial, the EMG trace was rectified and the area under the curve corresponding to the MEP was calculated. For both recorded muscles (OP/FDI), the mean MEP area in each experimental condition (type of stimulus and side) was expressed as a percentage of the grand mean.

A three-way analysis of variance (ANOVA) was performed on these data with affordance (normal, broken or absent), side (left or right) and recorded muscle (OP/FDI) as within-subjects factors. The significance level was set at $p<0.05$. When required, post hoc analyses were performed using LSD Fischer protected $t$-tests. A Mauchly test showed that the sphericity assumption was not violated.

\section{Results}

The ANOVA showed no significant effect of affordance $\left(F_{(2,22)}=0.08\right)$, side $\left(F_{(1,11)}=2.01\right)$ nor muscle $\left(F_{(1,11)}=0.67\right)$. Only the interaction between affordance and side $\left(F_{(2,22)}=4.66, p=0.02-\right.$ see Fig. 2) was significant. Post hoc analysis revealed that the interaction was due to the fact that, for the normal handle, the mean MEP area was larger when the handle was located to the right side than to the left side ( $111 \%$ vs. $89 \%, p<0.003$ ) while such a modulation was not found for the broken handle (97\% vs. $100 \%$, $p=0.61)$ nor for the control condition ( $101 \%$ vs. $101 \%, p=0.98)$. Moreover, for the right normal handle the mean MEP area was larger than for the right broken handle (111\% vs. 97\%, $p<0.04)$. Importantly, the fact that the interaction between the three factors was not reliable $\left(F_{(2,22)}=0.13\right)$ indicates that this interaction was present for both OP and FDI muscles. The remaining interactions between affordance and muscle $\left(F_{(2,22)}=0.61\right)$ and between side and muscle $\left(F_{(1,11)}=0.09\right)$ were not significant.

\section{Discussion}

The following discussion will consist of two parts: in the first we will discuss the meaning of the present results in motor terms and how they may contribute to better defining the interactions between ventral and dorsal stream; in the second part we will consider how the present results may contribute to ruling out the role of asymmetrical aspects of an object per se in determining the modulation of motor system activity.

\subsection{Affordances and the dorsal stream}

The present data clearly show that motor programs for grasping objects are specifically related to some specific pragmatic features of objects like, for example, handle orientation. In fact 
MEPs recorded from the right-hand were significantly larger when objects were presented with a normal handle on the right side versus when the handle was presented on the opposite side. Brain imaging studies have shown that the dorsal stream, and specifically its parietal sector, is sensitive to the changes in the orientation of graspable objects (Rice, Valyear, Goodale, Milner, \& Culham, 2007; Valyear, Culham, Sharif, Westwood, \& Goodale, 2006). The present study further supports this finding, showing that the primary motor area reflects the modulation of activity in parieto-premotor circuits, from which it receives connections (Rizzolatti \& Luppino, 2001), during the actual or potential execution of action.

Further, our data show that the recruited motor programs are fine tuned to privileged components so that as soon as these are violated, the related motor programs are consequently interrupted, as testified by MEPs recorded when objects were presented with the broken handle on the right side. These results are further supported by the lack of MEP differences in the condition of absent affordances, where no object is shown. Consistent with this, in an fMRI study Valyear, Cavina-Pratesi, Stiglick, and Culham (2007) found an activation within a parietal sector of the dorsal stream, distinct from that related to grasping, during the naming of tools. The authors have suggested that this activity may be related to skillful use of familiar objects.

One may argue of course that a cup can be grasped even if the handle is broken. If this was a determining factor we should expect a recruitment of the motor system in the broken handle condition, revealed by an increase in MEP area possibly related to alternative affordances like the top, the body or even the broken handle of the cup. The present data, however, clearly show that no difference was present between right and left when the handle was broken (as well as in the control condition). This suggests that, at least with stimuli used in the present experiment, the lack of modulation of the primary motor cortex possibly reflects the lack of recruitment of motor representations normally related to the skilful use of familiar objects. These results were obtained $200 \mathrm{~ms}$ after the presentation of the used stimuli.

In our opinion the present findings allows us to forward a different hypothesis about the roles of the dorsal and ventral streams as compared to the well-accepted view of the dorsal and ventral stream in the guidance of action and in building of percepts (Milner \& Goodale, 1995; Ungerleider \& Mishkin, 1982). According to the latter view the ventral stream is involved in the conceptual knowledge of objects ("what"), while the dorsal stream is involved in visuomotor transformations ("where" and "how"). According to Milner and Goodale (2008) "the key contribution of the ventral stream is the identification of possible and actual goal objects and the selection of an appropriate course to deal with those objects. The implementation of the constituent movements forming those actions and the on-line control is the role of the dorsal stream". This is the specialized meaning that authors give to vision for action: not the use of visual information for abstract planning but rather its use in the detailed programming and real time control at the level of elementary movements. If this were the only and specific role of the dorsal stream in sensori-motor transformation, one should expect a recruitment of this system even in the absence of a whole handle. Clearly this is not the case because alternative programs related to elementary movements were not recruited in the present study. What is the meaning of this lack of recruitment within the dorsal stream? The typical cup we have in mind is an object that can be handled and used to drink. However, this is a general concept which cannot be applied to any cup: some of them, for example, could be broken. How can we update our notion of cup? The dorsal stream could supply the information about the graspability of the object we have in mind, so that a broken cup is the cup we cannot grasp by handle. In this respect therefore the role of the dorsal stream could be not only the implementation of the detailed movements necessary to interact with a recognized object but the provision of online elements to update our notion of the actual cup we have to face. Although in a different field, data supporting this notion come from a recent fMRI study (Tettamanti et al., 2008) in which our ability to represent and understand the meaning of negative sentences was investigated. Participants had to passively listen to sentences defined by a factorial combination of polarity (affirmative vs. negative) and concreteness (action-related vs. abstract). Negative sentences were characterized by a decrease of activation with respect to affirmative sentences and, more importantly for our purposes, there was a specific reduction of activation for action-related negative sentences in left fronto-parieto-temporal areas or, in other words, in the action-representation system that is usually recruited in processing action related linguistic material (Buccino et al., 2005; Tettamanti et al., 2005; for a review see also Pulvermüller, 2005). This suggests that the subjective experience of negation may have as a neural counterpart the suppression or deactivation of the cortical representation of the action expressed in the sentence. In this respect the situation appears quite parallel to that of a cup with a broken handle. This cup cannot be grasped in the usual way and the deactivation of the motor system could be necessary for our understanding that a cup which cannot be grasped is a broken cup.

\subsection{The role of asymmetrical aspects of an object}

Recently, Phillips and Ward (2002) found an affordance effect related to a primed object regardless of the modality of the response: hands uncrossed, hands crossed or foot response. These authors interpreted their results assuming an abstract spatial response code that potentiates a wide variety of lateralized responses corresponding with the affordance. However, another explanation is possible. Primed stimuli used in the study of Phillips and Ward were everyday asymmetrical objects: in fact the presence of a handle makes the objects asymmetric. This is also true for most studies concerning the role of pragmatic features of objects in the modulation of the motor system (e.g. Grèzes et al., 2003; Tucker \& Ellis, 1998). This asymmetry could make some parts of the object more salient than others thus capturing attention on the location of the more salient part. This has lead Anderson, Yamagishi, and Karavia (2002) to criticize the notion that affordances effect is linked to automatic processing of object pragmatic features. Alternatively these authors suggested that this is a consequence of the way visual attention is oriented to the most salient object part thus leading to the activation of coherent responses. In keeping with this approach Anderson et al. (2002) used both symmetric and asymmetric objects and no objects. Objects were presented centrally but were inclined rightwards or leftwards. Participants had to judge whether the orientation of the objects were clockwise or anticlockwise. RTs were faster in relation to the asymmetric component both for objects and no objects. The results of the present study are clearly against the hypothesis that the asymmetrical aspects of an object per se may recruit a corresponding motor program. Since the stimuli we used were asymmetrical in spite of the fact that the handle was broken (see Fig. 1), this result cannot be explained without referring to the pragmatic role of the handle. On the other hand the present results fit with the premotor theory of attention (Rizzolatti, Riggio, Dascola, \& Umiltà, 1987; Rizzolatti, Riggio, \& Sheliga, 1994) which proposes that spatial attention derives from the activation of visuomotor circuits that transform visual information into action.

In conclusion, our data suggest a more active role than previously assumed for the dorsal stream in extracting pragmatic features of objects and a possible role of this information in building up and updating our object knowledge. Further they do not seem to support any role of asymmetric features of objects in the recruitment and modulation of the motor system during perception for action. 


\section{Acknowledgments}

We would like to thank Patricia Gough for language revision.This work was supported by the VolkswagenStiftung and the FP7 project ROSSI, Emergence of communication in RObots through Sensorimotor and Social Interaction, Grant agreement no: 216125.

\section{References}

Anderson, S. J., Yamagishi, N., \& Karavia, V. (2002). Attentional processes link perception and action. Proceedings of the Royal Society of London B, 269, 1225-1232.

Binkofski, F., Buccino, G., Posse, S., Seitz, R. J., Rizzolatti, G., \& Freund, H. J. (1999). A fronto-parietal circuit for object manipulation in man: Evidence from an fMRIstudy. European Journal of Neuroscience, 11, 3276-3286.

Buccino, G., Riggio, L., Melli, G., Binkofski, F., Gallese, V., \& Rizzolatti, G. (2005). Listening to action-related sentences modulates the activity of the motor system: A combined TMS and behavioral study. Cognitive Brain Research, 24, 355-363.

Chao, L. L., \& Martin, A. (2000). Representation of manipulable man-made objects in the dorsal stream. NeuroImage, 12, 478-484.

Craighero, L., Fadiga, L., Rizzolatti, G., \& Umiltà, C. (1999). Action for perception: A motor-visual attentional effect. Journal of Experimental Psychology: Human Perception and Performance, 25, 1673-1692.

Ellis, R., \& Tucker, M. (2000). Micro-affordance: The potentiation of components of action by seen objects. British Journal of Psychology, 91, 451-471.

Fischer, M. H., \& Dahl, C. (2007). The time course of visuo-motor affordances. Experimental Brain Research, 176, 519-524.

Gibson, J. J. (1977). The theory of affordances. In R. Shaw, \& J. Bransford (Eds.), Perceiving, acting and knowing (pp. 67-82). Hillsdale, NJ: Erlbaum.

Gibson, J. J. (1979/1986). The ecological approach to visual perception. Boston: Houghton-Mifflin.

Grafton, S. T., Fadiga, L., Arbib, M. A., \& Rizzolatti, G. (1997). Premotor cortex activation during observation and naming of familiar tools. NeuroImage, 6, 231236

Grèzes, J., Tucker, M., Armony, J., Ellis, R., \& Passingham, R. E. (2003). Objects automatically potentiate action: An fMRI study of implicit processing. European Journal of Neuroscience, 17, 2735-2740.

Jeannerod, M. (1995). Mental imagery in the motor context. Neuropsychologia, 33, $1419-1432$.

Mills, K. R., Boniface, S. J., \& Schubert, M. (1992). Magnetic brain stimulation with a double coil: The importance of coil orientation. Electroencephalography and Clinical Neurophysiology, 85, 17-21.

Milner, A. D., \& Goodale, M. A. (1995). The visual brain in action. Oxford: Oxford University Press.

Milner, A. D., \& Goodale, M. A. (2008). Two visual systems re-viewed. Neuropsychologia, 46, 774-785.

Murata, A., Fadiga, L., Fogassi, L., Gallese, V., Raos, V., \& Rizzolatti, G. (1997). Object representation in the ventral premotor cortex (area F5) of the monkey. Journal of Neurophysiology, 78, 2226-2230.

Oldfield, R. C. (1971). The assessment and analysis of handedness: The Edinburgh inventory. Neuropsychologia, 9, 97-113.
Phillips, J. C., \& Ward, R. (2002). S-R correspondence effects of irrelevant visual affordance: Time course and specificity of response activation. Visual Cognition, 9, $540-558$.

Pulvermüller, F. (2005). Brain mechanisms linking language and action. Nature Reviews Neuroscience, 6, 576-582.

Rice, N.J., Valyear, K. F., Goodale, M. A., Milner, A. D., \& Culham, J. C.(2007). Orientation sensitivity to graspable objects: An fMRI adaptation study. NeuroImage, 36(Suppl. 2), 87-93.

Rizzolatti, G., Riggio, L., Dascola, I., \& Umiltà, C. (1987). Reorienting attention across the horizontal and the vertical meridians: Evidence in favor of a premotor theory of attention. Neuropsychologia, 25, 31-40.

Rizzolatti, G., Camarda, R., Fogassi, L., Gentilucci, M., Luppino, G., \& Matelli, M. (1988) Functional organization of inferior area 6 in the macaque monkey. II. Area F5 and the control of distal movements. Experimental Brain Research, 71, 491-507.

Rizzolatti, G., Riggio, L., \& Sheliga, B. M. (1994). Space and selective attention. In C. Umiltà, \& M. Moscovitch (Eds.), Attention and performance XV: Conscious and nonconscious information processing (pp. 231-265). Cambridge, MA: MIT Press.

Rizzolatti, G., \& Luppino, G. (2001). The cortical motor system. Neuron, 31, 889-901.

Rossini, P. M., Barker, A. T., Berardelli, A., Caramia, M. D., Caruso, G., Cracco, R. Q., et al (1994). Non-invasive electrical and magnetic stimulation of the brain, spinal cord and roots: Basic principles and procedures for routine clinical application. Report of an IFCN committee. Electroencephalography and Clinical Neurophysiology, 91 79-92.

Sakata, H., Taira, M., Murata, A., \& Mine, S. (1995). Neural mechanisms of visual guidance of hand action in the parietal cortex of the monkey. Cerebral Cortex, 5 , 429-438.

Tettamanti, M., Buccino, G., Saccuman, M. C., Gallese, V., Danna, M., Scifo, P., et al. (2005). Listening to action-related sentences activates fronto-parietal motor circuits. Journal of Cognitive Neuroscience, 17, 273-281.

Tettamanti, M., Manenti, R., Della Rosa, P. A., Falini, A., Perani, D., Cappa, S. F., et al. (2008). Negation in the brain: Modulating action representations. Neurolmage 43, 358-367.

Tucker, M., \& Ellis, R. (1998). On the relations between seen objects and components of potential actions. Journal of Experimental Psychology: Human Perception and Performance, 24, 830-846.

Tucker, M., \& Ellis, R. (2001). The potentiation of grasp types during visual object categorization. Visual Cognition, 8, 769-800.

Tucker, M., \& Ellis, R. (2004). Action priming by briefly presented objects. Acta Psychologica, 116, 185-203.

Ungerleider, L. G., \& Mishkin, M. (1982). Two cortical visual systems. In D. J. Ingle, M. A. Goodale, \& R. I. W. Manfield (Eds.), Analysis of visual behaviour (pp. 549-586). Cambridge, MA: MIT Press.

Valyear, K. F., Culham, J. C., Sharif, N., Westwood, D., \& Goodale, M. A. (2006). A double dissociation between sensitivity to changes in object identity and object orientation in the ventral and dorsal visual streams: A human fMRI study. Neuropsychologia, 44, 218-228.

Valyear, K. F., Cavina-Pratesi, C., Stiglick, A. J., \& Culham, J. C. (2007). Does toolrelated fMRI activity within the intraparietal sulcus reflect the plan to grasp? NeuroImage, 36(Suppl. 2), 94-108.

Wassermann, E. M. (1998). Risk and safety of repetitive transcranial magnetic stimulation: Report and suggested guidelines from the international workshop on the safety of rTMS. Electroencephalography and Clinical Neurophysiology, 108, 1-16. 